\title{
MANAGING ALCOHOL CONSUMPTION - A RESPONSIBLE CONSUMPTION APPROACH
}

\author{
Weng Marc Lim, School of Business, Monash University \\ Ding Hooi Ting, School of Business, Monash University \\ Jia Jun Mah, School of Business, Monash University \\ Chee Yue Wong, School of Business, Monash University
}

dx.doi.org/10.18374/JIMS-13-2.8

\begin{abstract}
The purpose of this paper is to understand the perception of consumers towards alcohol consumption and provide recommendations to promote the responsible consumption of alcohol. A group of 28 people age between 18-60 years of different religions except Muslim was interviewed. Several factors were found to be the reasons for alcohol consumption, namely, applicability of alcohol consumption law only directed towards Muslims (i.e Syariah Law), family and occasion influences, curiosity, and peer pressure. Perceptions on binge drinking were not only viewed as a health risk, but also as a way out of problems. Knowledge on health benefits and harms of alcohol consumption was also observed to be shallow. Moreover, underage drinking was found to be common and is supported by families and occasions throughout the growing up years of consumers in Malaysia. Unlike existing studies which focused on the perceptions and dangers of alcohol consumption in developed countries, this qualitative paper is first of its kind as it uncovers the reasons for alcohol consumption in a developing country (i.e. Malaysia), and perceptions of consumers towards binge drinking, knowledge on health benefits and harms of alcohol consumption, and underage drinking to rationalize the extent to which alcohol consumption is considered to be a responsible one.
\end{abstract}

Keywords: Responsible Consumption, Alcohol 